\title{
Comparative study of chemical synthesis of dihydropyrimidine (DHPMS) derivatives by Biginelli Reaction using microwave irradiation and conventional method
}

\author{
Ratnamala P. Sonawane \\ The Institute of Science, Mumbai 400032, India \\ Email address: ratnasonawane@yahoo.in
}

\begin{abstract}
The majority of the drugs on the market today are entirely chemically synthesized in the laboratory. Several scientists had synthesized dihydropyrimidine (DHPMs) derivatives showing a wide spectrum of biological actions as antibacterials, antivirals as well as antitumor agents. This activity is principally due to presence of steriogenic carbon $\mathrm{C} 4$ in their structure. The current investigation is comparative study of chemically synthesis of two DHPMs derivatives by Biginelli Reaction using microwave irradiation and conventional method. The synthesis of DHPMs derivative involves a multicomponent reaction (aldehyde derivative, urea /thiourea and 1,3-dicarbonyl compounds) in presence of $\mathrm{HCl} / \mathrm{NH}_{4} \mathrm{Cl}$ as a catalyst. Two derivative viz. 3,4-dihydropyrimidin-2$(1 \mathrm{H})$-ones and 3,4-dihydropyrimidin-2-(1H)-thiones were synthesized and characterized using IR. The melting points were obtained are $203{ }^{\circ} \mathrm{C}$ and $211^{\circ} \mathrm{C}$ respectively. Microwave irradiation was easy and gave more yield than conventional method. This study will help to develop easy protocol for the synthesis of many more DHPMs derivative with high yield.
\end{abstract}

Keyword: dihydropyrimidine derivatives; Biginelli Reaction; microwave irradiation; conventional method

\section{INTRODUCTION}

The original one pot synthesis of 3,4-dihydropyrimidine-2- $(1 \mathrm{H})$ - ones was firstly reported by Pietro Biginelli in 1893 performing the three component cyclocondensation reaction of ethyl acetoacetate, benzaldehyde and urea under Bronsted acid catalysis ${ }^{1-2}$. However this reaction suffers from the harsh conditions, high raction times and frequently low yields Aryl substituted 3,4-dihydropyrimidine-2- $(1 \mathrm{H})-$ ones $^{3}$ and their derivatives are an important class of substances in organic and medicinal chemistry.

Many synthetic methods ${ }^{4-8}$ for preparing these compounds have been reported including classical methods, microwave irradiation and by using Lewis acids as well as protic acids.

The discovery of a new an inexpensive catalyst for the preparation of 3,4dihydropyrimidine-2-(1H)- ones under neutral and mild conditions with high yield of prime importance. In solvent free organic reactions ${ }^{9}$ reagents react together in the absence of any solvent have been reviewed as a fast developing technology. 


\section{EXPERIMENTAL}

The chemicals used Benzaldehyde, Ethyl acetoacetate, urea, thiourea, methanol, ethanol, ammonium chloride, hydrochloric acid were of analytical reagent grade. Methods used for synthesis of 3,4-dihydropyrimidine-2-(1H)- ones and their derivativesare conventional method and microwave method. Melting points were determined in open capillary tubes on a Buchi 530 melting point apparatus. The IR spectra were recorded on Shimadzu 435 FT- IR spectrophotometer with samples prepared as KBr palletes.

\section{1. Synthesis of 5-ethoxycarbonyl-4-phenyl-6-methyl-3,4-dihydropyrimidine-2-(1H)- one/thiones by conventional method}

A mixture of benzaldehyde $(1 \mathrm{mmol})$, ethyl acetoacetate $(1 \mathrm{mmol})$, urea/ thiourea $(1 \mathrm{mmol})$ and catalytic amount of $\mathrm{HCl} / \mathrm{NH}_{4} \mathrm{Cl}$ was taken in round bottom flask with $30 \mathrm{~cm}^{3}$ methanol and reflux at $100{ }^{\circ} \mathrm{C}$ for $3 \mathrm{hrs}$. The progress of the reaction was monitored by TLC.

The mixture was cooled to room temperature and poured in cold water.the solid product was collected by filtration. The product was dried and recrystllised from hot alcohol to obtained the pure product. Product was characterized by melting point, 1H NMR, IR, Mass Spectra.

\section{2. Synthesis of 5-ethoxycarbonyl-4-phenyl-6-methyl-3,4-dihydropyrimidine-2-(1H)- one/thiones by microwave method}

A mixture of benzaldehyde $(1 \mathrm{mmol})$, ethyl acetoacetate $(1 \mathrm{mmol})$, urea/ thiourea ( $1 \mathrm{mmol}$ ) and catalytic amount of $\mathrm{HCl} / \mathrm{NH}_{4} \mathrm{Cl}$ was taken in a flask. It is then irradiated in a microwave oven $840 \mathrm{~W}$ for required duration. Mixture is cooled to room temperature and water was added with stirring. The solid product precipitated out which was filtered. The crude product was recrystallised from alcohol. Product was characterized by melting point, $1 \mathrm{H}$ NMR, IR, Mass Spectra.

\section{RESULTS AND DISCUSSION}

5-ethoxycarbonyl-4-phenyl-6-methyl-3,4-dihydropyrimidine-2-(1H)-one prepared was confirmed by IR spectra. Interpretations of these spectras were carried out in Table 1 and 2 . It was also confirmed by NMR spectra. (DMSO-d-6) d: 1.09 (t, 3H, J=7.1 Hz, $-\mathrm{OCH}_{2} \mathrm{CH}_{3}$ ) 2.25(s, 3H, $\left.\mathrm{CH}_{3}\right), 3.97\left(\mathrm{q}, 2 \mathrm{H}, \mathrm{J}=7.1 \mathrm{~Hz},-\mathrm{OCH}_{2}\right), 5.05(\mathrm{~d}, 1 \mathrm{H}, \mathrm{J}=2.15 \mathrm{~Hz},-\mathrm{CH}), 7.28(\mathrm{~m}, 5 \mathrm{H}$, Ar-H), $7.75(\mathrm{~s}, 1 \mathrm{H}, \mathrm{NH}), 9.20(\mathrm{~s}, 1 \mathrm{H}, \mathrm{NH})$. Melting point was observed at $213{ }^{\circ} \mathrm{C}$.

5-ethoxycarbonyl-4-phenyl-6-methyl-3,4-dihydropyrimidine-2- $(1 \mathrm{H})$-thione prepared was confirmed by IR spectra. Interpretations of these spectra's were carried out in Table 3 and 4 . Melting point was observed at $210^{\circ} \mathrm{C}$. 
Table 1. Interpretation of IR spectra of 5-ethoxycarbonyl-4-phenyl-6-methyl-3,4-dihydropyrimidine$2-(1 \mathrm{H})$-one by conventional method.

\begin{tabular}{|c|c|c|}
\hline IR range $\left(\mathbf{c m}^{-\mathbf{1}}\right)$ & Peak Intensity & Peak due to \\
\hline 3247.99 & Sharp & N-H Stretching \\
\hline 3116.80 & Sharp & Aromatic C-H Stretching \\
\hline 2980.56 & Sharp & Aliphatic C-H Stretching \\
\hline 1725.85 & Sharp & Ester \\
\hline 1702.16 & Sharp & C=C Stretching \\
\hline 1650.57 & Sharp & Asymmetric C-H bending \\
\hline 1465.18 & Sharp & C-O Stretching \\
\hline 1270.88 & Sharp & Mono substituted benzene \\
\hline 700.46 & Sharp &
\end{tabular}

Table 2. Interpretation of IR spectra of 5-ethoxycarbonyl-4-phenyl-6-methyl-3,4-dihydropyrimidine$2-(1 \mathrm{H})$-one by microwave method.

\begin{tabular}{|c|c|c|}
\hline IR range $\mathbf{( c m}^{-\mathbf{1}} \mathbf{)}$ & Peak Intensity & Peak due to \\
\hline 3245.07 & Sharp & N-H Stretching \\
\hline 3120.60 & Sharp & Aromatic C-H Stretching \\
\hline 2980.43 & Sharp & Aliphatic C-H Stretching \\
\hline 1726.19 & Sharp & Ester \\
\hline 1703.96 & Sharp & C=O Stretching \\
\hline 1665.04 & Sharp & C=C Stretching \\
\hline 1465.04 & Sharp & Asymmetric C-H bending \\
\hline 1313.89 & Sharp & C-N Stretching \\
\hline 1290.99 & Sharp & C-O Stretching \\
\hline 700.31 & Sharp & $\begin{array}{c}\text { Mono substituted } \\
\text { benzene }\end{array}$ \\
\hline
\end{tabular}


Table 3. Interpretation of IR spectra of 5-ethoxycarbonyl-4-phenyl-6-methyl-3,4-dihydropyrimidine$2-(1 \mathrm{H})$-thiones by conventional method.

\begin{tabular}{|c|c|c|}
\hline IR range $\left(\mathbf{c m}^{-\mathbf{1}}\right)$ & Peak Intensity & Peak due to \\
\hline 3330.38 & Sharp & N-H Stretching \\
\hline 2981.25 & Sharp & Cliphatic C-H Stretching \\
\hline 1673.67 & Sharp & C=C Stretching \\
\hline 1575.38 & Sharp & Asymmetric C-H bending \\
\hline 1466.42 & Sharp & C=N Stretching \\
\hline 1371.61 & Sharp & C=S Stretching \\
\hline 1284.56 & Sharp & C-O Stretching \\
\hline 1178.47 & Sharp & Mono substituted benzene \\
\hline 724.25 & Sharp & . \\
\hline
\end{tabular}

Table 4. Interpretation of IR spectra of 5-ethoxycarbonyl-4-phenyl-6-methyl-3,4-dihydropyrimidine2-(1H)-thiones by microwave method.

\begin{tabular}{|c|c|c|}
\hline IR range $\left.\mathbf{( c m}^{-\mathbf{1}}\right)$ & Peak Intensity & Peak due to \\
\hline 3329.99 & Sharp & N-H Stretching \\
\hline 2981.34 & Sharp & Cliphatic C-H Stretching \\
\hline 1674.51 & Sharp Stretching \\
\hline 1575.70 & Sharp & C=C Stretching \\
\hline 1467.04 & Sharp & Asymmetric C-H bending \\
\hline 1371.62 & Sharp & C=N Stretching \\
\hline 1284.69 & Sharp & C=S Stretching \\
\hline 1120.61 & Sharp & C-O Stretching \\
\hline 724.32 & Sharp & Mono substituted benzene \\
\hline
\end{tabular}




\section{CONCLUSION}

The preparation of 5-ethoxycarbonyl-4-phenyl-6-methyl-3,4-dihydropyrimidine-2$(1 \mathrm{H})$-one/ thione by conventional and microwave assisted synthesis shows that by comparing the yield obtained with respect to time, the product obtained by microwave synthesis is in good yields and in less time and also avoids problems associated with solvent use.

The microwave synthesis is eco friendly. Solvent free microwave approach opens up numerous possibilities for conducting rapid organic synthesis and functional group transformations more efficiently. Additionaly there are distinct advantages of these solvent free reactions. It prevents pollution in organic synthesis at source. The chemo, regio, stereoselective synthesis of high value chemical entities and parallel synthesis to generate a library of small molecules will add to the growth of microwave enhanced reactions in the future.

\section{References}

[1] P. Biginelli, Chem Ber 24 (1891) 1317.

[2] F. Makaev, E. Styngach, V. Muntyanu, S. Pogrebnoi, Russian Journal of Organic Chemistry 43 (2007) 1512-1515.

[3] A. Shaabani, A. Bazgir, F. Teimouri, Tetrahedron Letters 44 (2003) 857-859.

[4] S. V. Ryabukhin, A. S. Plaskon, E. N. Ostapchuk, D. M. Volochnyuk., Synthesis 03 (2007) 417-427.

[5] I. Cepanec, M. Litvic, A. Bartolincic, Tetrahedron 61 (2005) 4275-4280.

[6] J. H. Schauble, E. A. Trauffer, P. P. Deshpande, R. D. Evans, Synthesis 08 (2005) 1333-1339.

[7] H. Hazarkhani, B. Karimi, Synthesis (2004) 1239-1242.

[8] J. Lu, Y. Bai, Synthesis (2002) 466-470.

[9] Tom Welton, Green Chem 8 (2006) 13. 